\title{
Infrared thermography applied to spontaneous combustion monitoring of coal tips
}

\author{
by O. Carpentier, D. Defer, E. Antczak, B. Duthoit
}

\author{
Université d'Artois \\ Laboratoire d'Artois Mécanique Thermique Instrumentation \\ Technoparc Futura \\ 62400 Béthune
}

\begin{abstract}
Spontaneous combustion of coal tips can generate dangerous slips and it is necessary to monitor this phenomena. Aerial IR thermography is one of methods widely used but, in the case of coal tips, it appears costly and not adapted to survey the evolution of reaction. It is for these reasons that the LAMTI in liaison with Charbonnages de France Group develops a method using topographic, thermographic and atmospheric survey that is intended to be more reliable and less expensive.
\end{abstract}

\section{Introduction}

Coal tips are conical or truncated-cone hills formed by piling up the waste from mining operations. Some of them still possess $5-15 \%$ of residual coal, the percentage varying according to the sorting techniques used. The oldest tips thus often contain more residual coal. The porous nature of the tip enables air and water to circulate, causing the coal to oxidise. Any iron sulphides such as marcassite and pyrites present in the tip will tend to catalyse the exothermal oxidation reaction, causing spontaneous combustion of the residual coal [1]. $10 \%$ of coal tips are undergoing a process of spontaneous combustion, the effects of which are as follows [2], slipping of the tip, accompanied by clouds of inflammable dust, formation of cavities, emission of noxious gases, creation of water-gas, a compound that explodes in air. Most failures occur during heavy rains, with penetrating water having the following additional direct effects (increase in the density of the materials, drop in shear strength, increase in pore pressures, downward movements, creating destabilising forces).

The effects can be particularly catastrophic. Slips are not classic rotational ones but, on account of the high water content in the schists, are more similar to mud slides; these can be deadly, as experienced in Virginia in the 1940s. There is also the added problem of major atmospheric pollution due to the release of sulphurous fumes [3]. As it is very expensive to fight coal tip fires, attempts have been made since the second half of the 20th century to prevent such fires during the formation of coal tips by taking precautions, such as for example not creating them above carbonate materials or spreading them out in order to reduce internal pressures. In the case of tips formed before this period, spreading them can be dangerous and costly if high-risk areas are not known, and monitoring then appears to be a necessary step.

\section{Cross reading topographic-thermographic surveys}

Combining thermography and topography means that it is possible to work over a large surface area and quickly. Once anomalies have been determined precisely, 
they can be monitored with regard to changes in temperature and displacement of the source. Information gathered in the field indicates the environmental conditions. Subsequently, the site can be monitored and it will be possible to tell whether temperature measurements indicate internal activity within the tip or are rather the direct result of environmental conditions.

Three stages are involved (figure 1). First, a topographic and thermographic survey in the field. Then we process the topographic data, pixels and infrared images to produce a $3 \mathrm{D}$ thermogram. Finally there is a post-processing of the temperatures, incorporating the environmental components.

An AGEMA 570 FPA monitoring camera was used with a resolution of 320x240 pixels working in the 8-14 $\mu \mathrm{m}$ wavelength band, which is suited to low temperatures (our field of work). The point set is gathered with a Trimble type GPS accurate to within a centimetre. The environmental data are collected with a meteo station with a data-taker. The topographic survey must be precise enough to offer a satisfactory reconstruction of the site so that the projection error between the images and the DTM is as small as possible. The thermal camera must be treated as both a monitoring tool and a theodolite. It is first necessary to carry out an investigation of the site as areas subject to slips can sometimes be identified with the naked eye (previous slips, fumes escaping, absence of vegetation). With regard to the actual monitoring, coal tips undergoing combustion generally provide good contrasts. However, work should not be carried out when it is raining as this tends to make surface temperatures uniform, or in strong sunlight, since the addition of atmospheric radiation is a source of error in surface temperatures. Lastly, low angles should be avoided, as the directional nature of emissivity means that its value decreases with distance from the normal angle of observation. The optimum conditions for measurements of this type are generally found at night as this is when the best contrast is obtained and the effect of atmospheric radiation is much lower. Surface temperature measurements made with a probe can nevertheless be used to correct the results obtained with a thermal camera. The raw data obtained in this way must therefore be processed, and this can only be done by collecting information on the site.

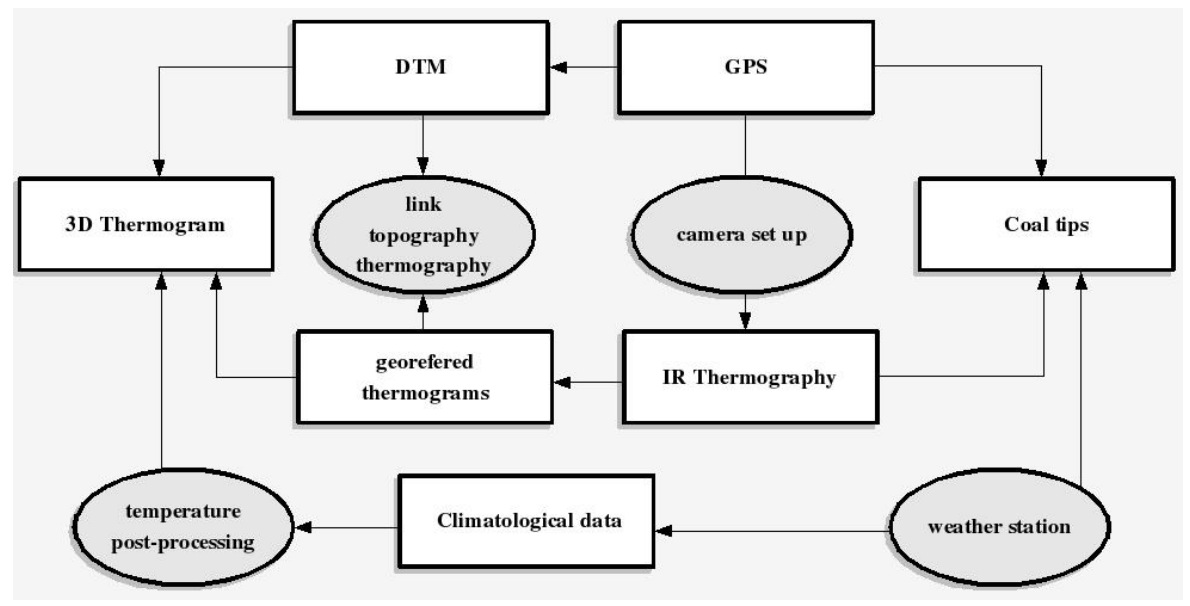

fig. 1. Combination of IR, GPS and climatological methods 


\section{Making a 3D thermogram}

In the area of research and application, the Digital Terrain Model (DTM) has become a common surveying technique for identifying forms of terrain. Infrared thermographic scanning creates specific geometric problems. Backing up imagery with topographic data (DTM) is a way of achieving a better understanding and higher degree of accuracy [4]. To prepare a 3D thermogram, a topographic method (GPS survey) is used with a view to forming a base for future modelling (figure 2). The location of coal tips and their simple nature overcomes the main problems arising with the GPS, namely multipath and mask phenomena. A GPS survey is collected in the form of a set of points. As the thermographic survey is combined with the topographic survey, it is possible to obtain georeferenced images and pixel coordinates can therefore be defined as $3 \mathrm{D}$ polar coordinates in relation to the camera, within a system chosen by the user (either national or local). As the images are georeferenced, they can be superimposed on the DTM (figure 3 ) by considering each pixel of each image taken during the monitoring survey. However, optical deformation by the camera lens introduces constant angular errors that distort the image in proportion to the distance from the central pixel. Part of the thermographic image must therefore be eliminated. The part to be eliminated will be determined by the tolerance applied to the angular distance in relation to an image that has not been distorted in any way. As an example, an error of one pixel at $100 \mathrm{~m}$ produces a difference of $13 \mathrm{~cm}$. A tolerance of $20 \mathrm{~cm}$ applied to the plotted points eliminates about $15 \%$ of the pixels from the image.

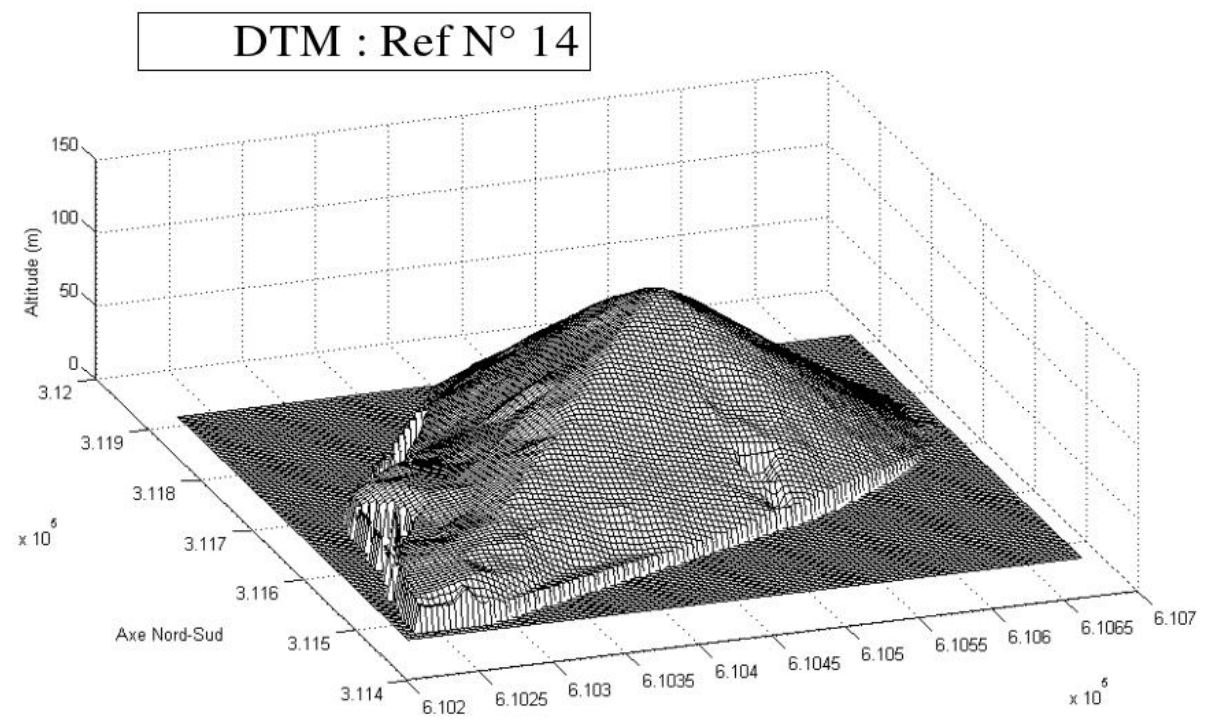

fig. 2. DTM composed by quadrangle elements 


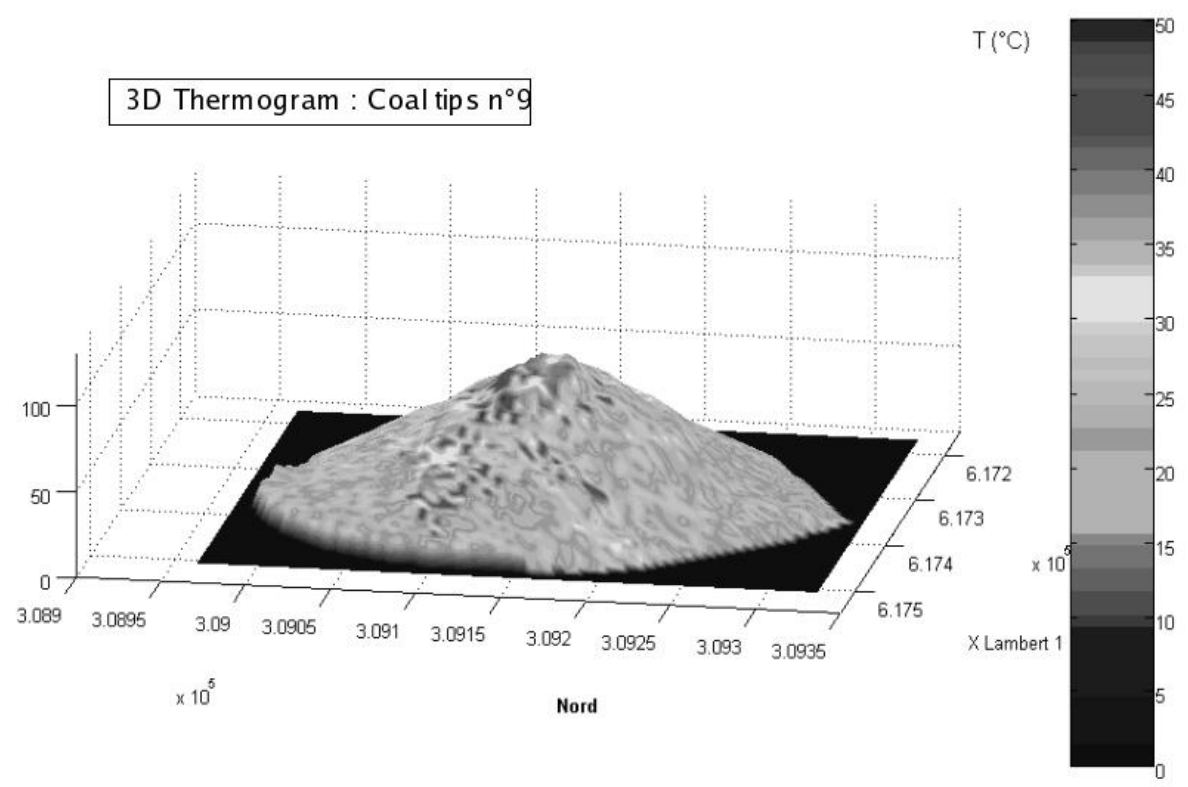

fig. 3. 3D thermogram - Slips areas on east side

\section{Post-processing}

Post-processing can take place after reconstruction, as the coordinates and temperatures of all the points are known. This means, for example, that several emissivity values can be selected from the same thermogram (coal tip, vegetation, etc.). The environment data must then be included. As the environment plays a predominant role in the in situ measurements, it was necessary to incorporate parameters such as the atmospheric transmission factor and the notion of thickness of the atmospheric layer into the measurement processes. Interface temperature modelling by FDM (Finite Difference Method) [5] allow us to estimate temperature evolution and weather conditions influences (figure 4). In this climatological approach, thermal balance of soil-atmosphere system is very important. Soil surface absorbs solar energy and infrared radiation form clouds and atmosphere. Heat losses are due to radiative exchanges to the atmosphere and convection. Finally, spontaneous combustion provides heat flow by conduction from source to surface. Flow summation correspond to vertical thermal balance of soil [6]. Measurements carried out with meteo station will allow us to estimate each flow in order to know the conductive flow only due to spontaneous combustion activity. This "absolute" flow, within weather condition influences can be used to monitor coal tips at different time of year and highlight the evolution of combustion from a thermal intensity point of view, but also if we consider the dimensional aspect thank to thermography measurement at the surface. 


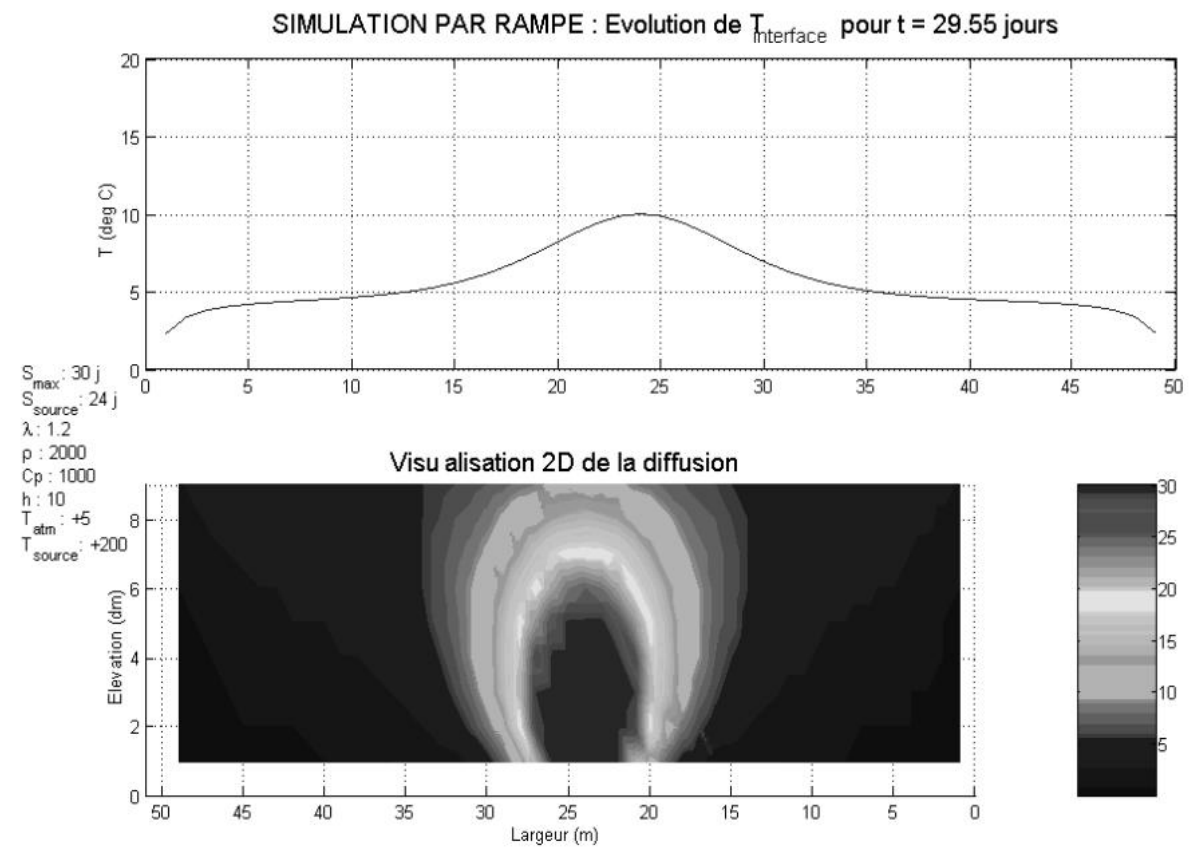

fig. 4. Source impact estimation by FDM

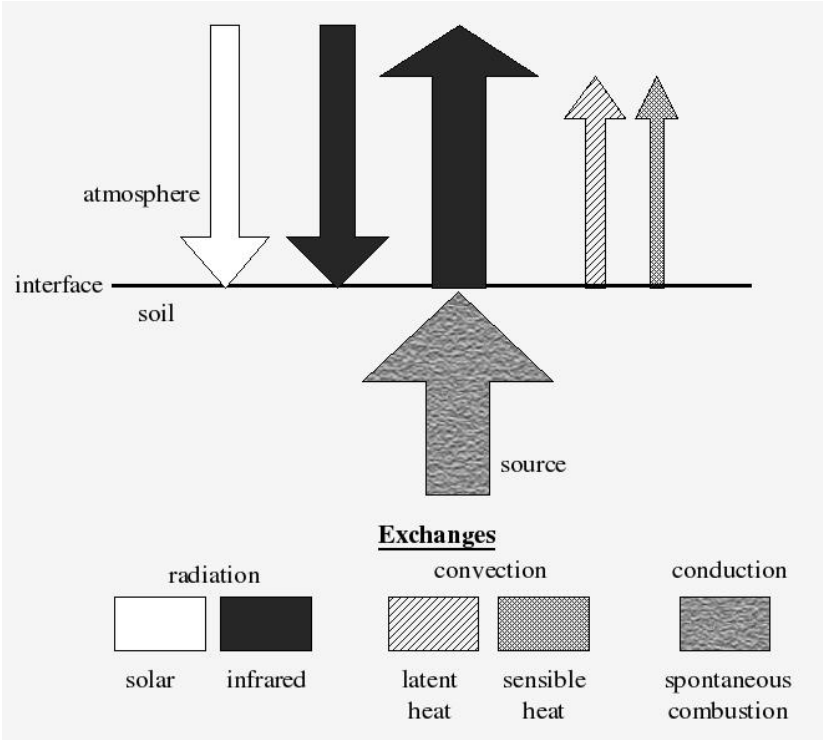

fig. 5. Flow having effect on thermal balance of soil-atmosphere system 


\section{Conclusion}

Ground-level infrared thermographic monitoring has the advantage of being more rigorous, more flexible and less costly than aerial monitoring surveys. Characterising the emissive character and modelling the micro-climate of the study environment are the keys for proposing a method for monitoring coal tip combustion reactions. It should be noted that an automatic system is currently being developed; this should enable the two methods to be linked without there being any need for the processes to be simultaneous, and should also minimise the need for operators in the field or even eliminate it if reconstruction is done on the basis of existing topographic data.

\section{REFERENCES}

[1] Harrington D., East J. H Incendies des terris, Annales des mines de Belgique, Tome 48, P. 427-434 (1948)

[2] Monjoie A., Schrodoer C. Instabilité des versant de terrils en relation avec l'autocombustion du charbon résiduel, Revue Française de Géotechnique, $\mathrm{N}^{\circ}$ 95-96, P. 91-102 (2001)

[3] Hebley F.H., Lecomte La lutte contre les feux de terrils, Annales des mines de Belgique, Tome 48, P. 709-712 (1948)

[4] Jupp D-L-B. Directional radiance and emissivity measurement models for remote sensing of the surface energy balance, Environmental Energy \& Software (1998)

[5] Ozisik N., Finite Difference Methods in heat transfert, Ed CRC Press (1994)

[6] Hufty, Introduction à la climatologie, Ed de Boeck, (2001) 